\title{
Bit Error Rate Performance Analysis of a Multiple Amplify and Forward Relaying Aided Cooperative MIMO-OFDM System
}

\author{
Md Mirazur Rahman, Md. Abdullah-Al-Mamun, Rizal Fathoni Kabir, Abidur Rahman, \\ Subaha Mahmuda
}

\begin{abstract}
In wireless systems the orthogonal frequency division multiplexing (OFDM) is one of the most influential means of transmission techniques. Data channel having higher speed are converted into some parallel channels by OFDM. For better performance of wireless network, cooperative communication using multiple relay is widely used. Substantial improvement can be achieved in case of reliability and throughput for wireless network if multiple relays are implemented between the source and destination by developing cooperative communication with multiple input multiple output (MIMO) OFDM. A mathematical model has been developed based on amplify and forward (AF) multiple relay for the system of cooperative MIMO-OFDM. Along with the detection system based on minimum mean squared error, the cooperative MIMO-OFDM, with multiple relays, can provide better performance than transmission system using single relay, which can be found from analyzing simulation. A considerable amount of deterioration was also noticed in the color image with changing the number of relays. Signal salvation is better for multiple relay than single relay.
\end{abstract}

Keywords: Multiple input multiple output, Bit error rate, Orthogonal frequency division multiplexing, Amplify and forward, Minimum mean square error and Zero forcing.

\section{INTRODUCTION}

The demand for enhanced internet services and wireless multimedia systems are escalating these days. Because of this, researchers are emphasizing on designing various wireless communications systems in view of fulfilling the ongoing and future demands. Among many cost effective, reliable and fast systems, Cooperative MIMO OFDM system

Revised Manuscript Received on February 14, 2020.

* Correspondence Author

Md Mirazur Rahman, Dept. of Electrical and Electronic Engineering, Primeasia University, Dhaka-1213, Bangladesh. Email: mrzmist@gmail.com

Md. Abdullah-Al-Mamun, Dept. of Electrical and Electronic Engineering, Ahsanullah University of Science and Technology, Dhaka-1208, Bangladesh. Email: mamunahsanullah@gmail.com

Rizal Fathoni Kabir, Dept. of Electrical, Electronic and Communication Engineering, Military Institute of Science and Technology, Dhaka-1216, Bangladesh. Email: rizalkabir@gmail.com

Abidur Rahman*, Dept. of Electrical and Electronic Engineering, Ahsanullah University of Science and Technology, Dhaka-1208, Bangladesh. Email: abid.eee@aust.edu

Subaha Mahmuda, Dept. of Electrical and Electronic Engineering, Primeasia University, Dhaka-1213, Bangladesh. Email: subaha2007@yahoo.com

(C) The Authors. Published by Blue Eyes Intelligence Engineering and Sciences Publication (BEIESP). This is an open access article under the CC BY-NC-ND license (http://creativecommons.org/licenses/by-nc-nd/4.0/) is an appropriate solution so far. For the simplification of implementation, fast Fourier transform is used which is a good choice of OFDM. A MIMO system incorporates multiple input multiple output technology by combining multiple antenna to ensure enhanced bit rate at receiver's end [1], and a cooperative system involves multiple number of nodes to form a virtual array of antenna [2]. That's why the diversity of the transceiver is minimized. Since it has good spectral efficiency, it can be used for advanced local area network and mobile broadband network standards. This approach is especially effective in using diversity gain by involving number of relays into operation, which is extremely useful to mitigate channel fading [3]. Additionally, this system promotes the low usage of power during transmission and improves the spatial diversity gain [1].

Several types of strategies can be taken for relaying including Amplify and forward (AF), decode and forward (DF), fixed relaying (FR), selection relaying (SR), coded cooperation (CC) and compress and forward (CF) as found in respectively [4-7]. Fixed relaying scheme is the simplest one, where information is continuously forwarded through the relay nodes [8]. However, the relay nodes remain inactive in the case of selective relaying unless a definite margin is exceeded by the channel [9]. Again, in the mode of incremental relaying, respond is made by relaying node once the receiving end requests [10]. There was an ongoing dispute on performances of two popular relaying protocols: AF and DF, recently. In [11], AF with multi-hop relaying exhibited better BER and outage probability than DF. However, in [12], for an encoded BPSK system, DF with multihop relaying has shown half of diversity compared to that of AF protocol.

Overall, the whole relay system can be divided into two parts: single relay and multiple relay system. Previously it was demonstrated that in terms of network reliability and transmission energy multiple-relay system can outperform a single relay system without affecting the BER. [13]. A clear study has been demonstrated in [7]-[14], where performance on text message transmission was evaluated based on a single relay cooperative OFDM system under AF and DF. Besides, [15] has shown that BER was considerably improved by using multiple relays with low SNR value for audio and text data. Based on current wireless standards, such as IEEE 802.16j, in [8-16] an OFDM based multi-hop relaying system has been proposed. The issues with implementation of cellular network based on OFDM-based multi-hop was accounted by Can et al. [17]. 


\section{Bit Error Rate Performance Analysis of a Multiple Amplify and Forward Relaying Aided Cooperative MIMO-OFDM System}

This study proposes a mathematical and simulation model of cooperative MIMO-OFDM system with multiple number of relays. Two linear detectors are considered: linear zero forcing (ZF) detector and linear minimum mean square error (MMSE) detector, where both are used to lessen the complexity of the receiver. Bit error rate (BER) and color image were set as performance markers based on which the performance of the whole system was measured.

\section{SYSTEM MODEL}

Fig. 1 shows a cooperative MIMO-OFDM communication system with $M(1,2$ and 4$)$ relays. The number of users in the system are $M+1$, one of whom is acting as a source and while other $\mathrm{M}$ users play the roles of relays.

There is no restriction of interchanging the message or information. At different instants of time, the $\mathrm{M}+1$ users can interchange their message or information as source and relay. Data error should be protected while simulating the system. For this reason, the original signal is being transmitted with a redundant data source. Thus, it works as a protection against data error in the simulated system. A simple convolution encoder having rate of $1 / 2$ is implemented in the coding scheme of the channel to provide the basis of such requirements. From the image signal, at first the data which is in binary form is extracted. These data are channel coded and interleaved subsequently. For the conversion of the binary data into such type of signal which is modulated digitally, BPSK is used. When the process of digital modulation is done, the complex digitally modulated data are rearranged and then set for different antennas. There is a converter available for each transmitting antenna section which receives data serially and delivers parallelly. All the data are passed via the converter. Then according to inverse fast Fourier transform (IFFT) these data are transformed into signal in time domain. Then a cyclic prefix (CP) is added. This is nothing but a copy of the elements that are in the last input of serial to parallel converter. While transmitting from each antenna, the informative symbols are transmitted at one time. This simultaneous transmission is done after the converter which converts parallel to serial. The signals are received in two phases at the receiving end. Phase I which is direct link and phase II which is relay link. While transferring, not only from source to destination but also from source to relay, the data are transferred directly in phase I. The data are transferred in the case of phase II from relay to destination. Then the received signal is amplified by each cooperative relay. Then for the destination, the amplified signal is forwarded via a wireless channel. At the destination end, an OFDM demodulator is used. After receiving the signals, they are passed through the demodulator. Then after digital demodulation, they are passed through de-interleaver and decoder sections which decode channel. After this, the actual signal is regained which was in the form of the original image.

\section{THEORETICAL ANALYSIS OF MULTIPLE RELAYS}

The convolution encoder is fed with the binary data stream as inputs, after being extracted from image/audio signals. Considering the length, $d$ of the binary stream is $L_{a}$ where $d_{i} \in\{0,1\}$ and $i=0,1,2,3 \ldots, L_{a}$ is made doubled (2 $L_{a}$, ) by adding a redundant binary bit after channel encoding. BPSK scheme was used to modulate this data digitally and afterwards, it was reorganized by using STBC [20, 21]. Here, data are arranged for the first and second antennas as,

$$
X_{k}^{T x}=\left[X_{0}, X_{1}, X_{2}, \ldots \ldots \ldots X_{K-1}\right]
$$

Then symbols were converted from serial to parallel where number of symbols, $K=1024$. After that they are processed through OFDM. Here the OFDM block uses inverse fast Fourier transform to convert the signal into time domain, which is defined as,

$$
x^{T x}(n)=\sum_{k=0}^{N_{C}-1} X_{k}^{T x} e^{j\left(\frac{2 \pi}{N_{C}}\right) k n}
$$

where, $N_{c}$ and $T_{x}$ indicate the number of subcarriers and transmission identifier respectively, and $j=\sqrt{ }(-1)$ is a complex number.

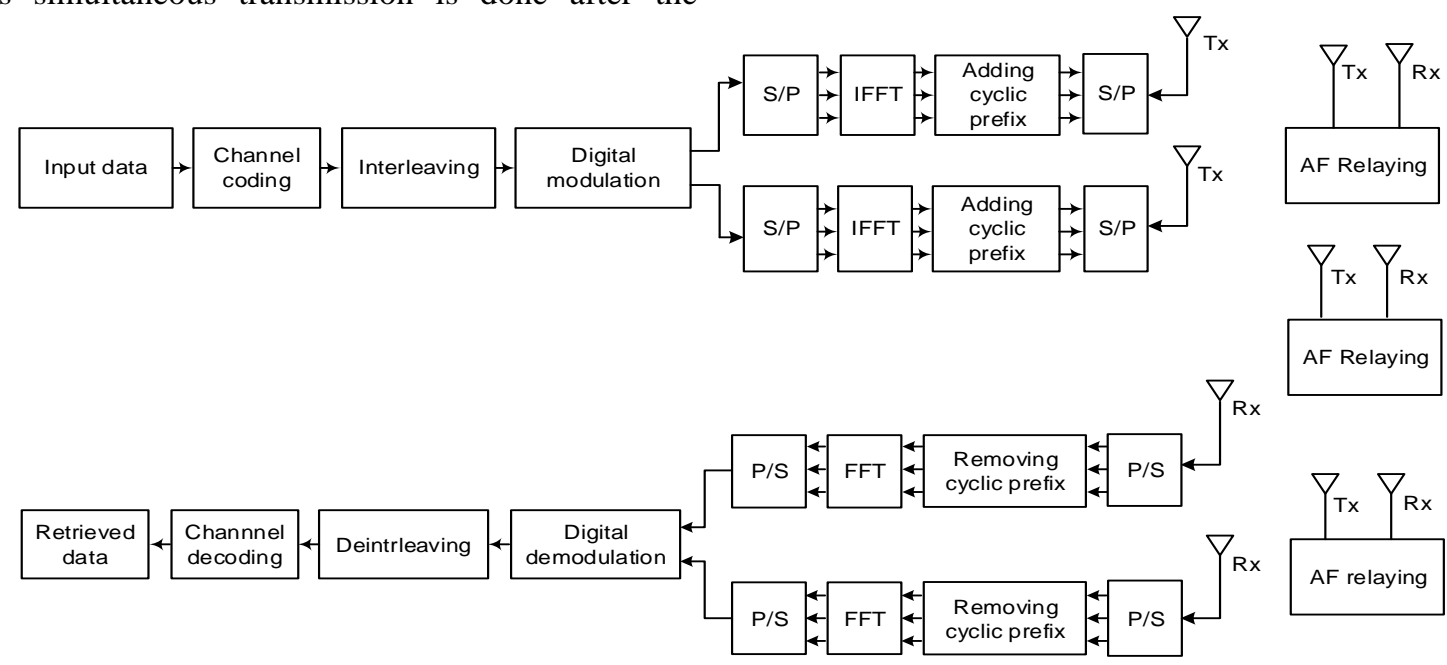

Fig. 1. Block diagram of Multiple Amplify and Forward Relayed Cooperative MIMO-OFDM system

Retrieval Number: C5727029320/2020@BEIESP DOI: 10.35940/ijeat.C5727.029320 Journal Website: www.ijeat.org 
The value of the integers, $n$ and $k$, varies between 0 and $N_{C}-1$. After adding with the cyclic prefix of length $N_{C P}$, the time domain discrete signal $X(n)$ can be defined as,

$$
x^{T x}(m)=\sum_{k=0}^{N_{C}+N_{C p}-1} X_{k}^{T x} e^{j\left(\frac{2 \pi}{N_{C}+N_{C P}}\right) k m}=\sum_{k=0}^{N-1} X_{k}^{T x} e^{j\left(\frac{2 \pi}{N}\right) k m}
$$

where $m=n+N_{C P}$ and $N=N_{C}+N_{C P}$

The source-destination (Equation 3) and source-relay (Equation 4) signals, for phase I, after these are sent through a discrete-time baseband channel are as follows

$$
\begin{aligned}
& r_{s, d}(m)=\sqrt{P_{s}}\left\{x^{T x}(m) \cdot h_{s, d}(m)+w_{d}^{1}(m)\right\} \\
& r_{s, r_{i}}(m)=\sqrt{P_{s}}\left\{S^{T x}(m) \cdot h_{s, r_{i}}(m)+w_{r_{i}}(m)\right\}
\end{aligned}
$$

where $h(m)$ denotes the response after passing through a discrete-time baseband channel and $w_{d}^{1}(m)$ and $w_{r i}(m)$ are the added additive white Gaussian noise (AWGN). Also, $r_{s, d}(m)$ and $r_{s, r}(m)$ stand for source to destination and source to relay signals respectively. In amplify-and-forward (AF) relay scheme, a signal is received from the source. After amplifying, the received signal is forwarded to the destination where quality of the source-relay link is disregarded.

A linear precoder $(F)$ matrix $\left(M_{s} \times M_{r}\right)$ is employed by the relay in Phase II on the source to relay signal vector $r_{s, r}(m)$ which can be expressed as [10-12, 18-20] where $\sigma_{r}^{2}$ denotes the variance of relay noise, and number of relay receiving and transmitting antennas are express as $M_{s}$ and $M_{r}$ respectively:

$$
F=\sqrt{\frac{1}{\operatorname{tr}\left(H_{s, r_{i}}^{H} H_{s, r_{i}}\left(\sigma_{r_{i}}^{2} I_{M_{r_{i}}}+\frac{P_{s}}{M_{s}} H_{s, r_{i}}^{H} H_{s, r_{i}}\right) H_{r_{i}, d} H_{r_{i}, d}^{H}\right.}} H_{s, r_{i}}^{H} H_{r_{i}, d}^{H}
$$

Additionally, channel matrices of the source to relay and relay to destination are referred as $H_{s, r}$ and $H_{r, d}$ respectively. Therefore, the transmitted signal from the relay can be expressed as,

$$
x_{r}(m)=F \cdot r_{s, r_{i}}(m)
$$

and this is further received at the destination in the form of,

$$
\begin{aligned}
r_{r, d}(m)= & {\left[\sqrt{P_{r} P_{s}} F x^{T x}(m) h_{s, r_{i}}(m)\right] h_{r_{i}, d}(m) } \\
& +\left[\sqrt{P_{r}} F w_{r_{i}}(m)\right] h_{r_{i}, d}(m)+w_{d}^{2}(m) \\
= & {\left[\sqrt{P_{r} P_{s}} F x^{T x}(m) h_{s, r_{i}}(m)\right] h_{r_{i}, d}(m)+\widetilde{w}_{d}^{2} }
\end{aligned}
$$

where, $P_{r}$ indicates the power of relayed signal, $\mathrm{w}(m)$ and $w_{r}(m)$ are the AWGN at destination and relay respectively. Also, $\widetilde{w}_{2}^{d}$ denotes the effective noise in Phase II. After adding the received signals of both phase (Phase I and II) at the destination we find,

$$
\begin{aligned}
r_{d-\text { combined }}(m) & =\left[\sqrt{P_{r} P_{s}} F x^{T x}(m) h_{s, r_{i}}(m)\right] h_{r_{i}, d}(m) \\
& +\widetilde{w}_{d}^{2}+\sqrt{P_{s}}\left\{x^{T x}(m) h_{s, d}(m)\right\}+w_{d}^{1}(m)
\end{aligned}
$$

This can be expressed in matrix form as following,

$$
\begin{aligned}
& r_{d-\text { combined }}(m)=\left[\begin{array}{l}
r_{s, d}(m) \\
r_{r, d}(m)
\end{array}\right] \\
& =\sqrt{P_{s}}\left[\begin{array}{c}
h_{s, d}(m) \\
\sqrt{P_{r}} h_{r_{i}, d}(m) F h_{s, r_{i}}(m)
\end{array}\right] x^{T x}(m) \\
& +\left[\begin{array}{l}
w_{d}^{1}(m) \\
\widetilde{w}_{d}^{2}(m)
\end{array}\right]
\end{aligned}
$$

here, the effective channel between source and destination,

$$
H(m)=\left[\begin{array}{c}
h_{s, d}(m) \\
\sqrt{P_{r}} h_{r_{i}, d}(m) F h_{s, r_{i}}(m)
\end{array}\right]
$$

and the effective noise will be,

$$
(m)=\left[\begin{array}{l}
w_{d}^{1}(m) \\
\widetilde{w}_{d}^{2}(m)
\end{array}\right]
$$

After implementing the equalization of channel, cyclic prefixing schemes are removed subsequently. Finally, the detected signal $r_{d}(n)$ is fed into the fast Fourier transform (FFT) section where the size of FFT is $N=N_{C}+N_{C P}$. Thus, the output obtained as,

$$
Y_{F F T}(k)=\frac{1}{N} \sum_{n=0}^{N-1} r_{d}(n) e^{-j\left(\frac{2 \pi}{N}\right) k n}
$$

\section{A. ZF Signal Detection}

Channel matrix is reversed by using Zero-forcing (ZF) detectors. By following weight matrix the Zero Forcing technique nullifies the interference $[13,14]$

$$
W_{Z F}=\left(H^{H} H\right)^{-1} H^{H}
$$

where $(.)^{H_{l}}$ denotes the Hermitian transpose operation. This means, it inverts the effect of channel as,

$$
x_{Z F}=W_{Z F} Y(k)
$$

\section{B. MMSE Signal Detection}

A linear filter is employed by MMSE detector which can consider the noise. The MMSE filter is found by minimizing the mean-square error as [21, 22],

$$
W_{M M S E}=\left(H^{H} H+\sigma_{N_{k}}^{2} I\right)^{-1} H^{H}
$$

By suing a SNIR, the MMSE detection converts to ZF detection. After using the MMSE weight in equation (13), we obtain the following relationship.

$$
X_{M M S E}=W_{M M S E} Y(k)
$$

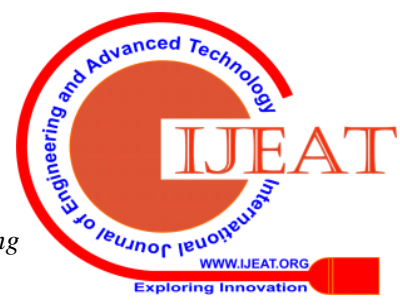




\section{Bit Error Rate Performance Analysis of a Multiple Amplify and Forward Relaying Aided Cooperative MIMO-OFDM System}

\section{RESULTS AND DISCUSSION}

In this section, the simulation results obtained from MATLAB are presented and discussed. Various number of relays can be used for transmitting the signal to compare the results. Single relay, two relays and four relays are used for the estimation of BER on colour image transmission by using computer simulation works. Half-rated Convolutional Encoder, BPSK, 1024 and 103 symbols are being set for the Channel Coding, Digital modulation, FFT/IFFT size and CP length respectively. $\mathrm{CP}$ is a key element of enabling the OFDM signal to operate reliably. It acts as a buffer region or guard interval to protect the OFDM signals from inter symbol interference.

We can observe from the Fig. 2 that a comparatively better system performance can be achieved under the situation if the relaying scheme is activated near the sender. The underlying cause of this better output is due to the fact that if the relay is situated comparatively near to the source then the degradation of signal from the source will be much less. We also observe the signal when the relay is further from the destination. Subsequently, the BER decreases when the proximity of the relay is near to the destination.

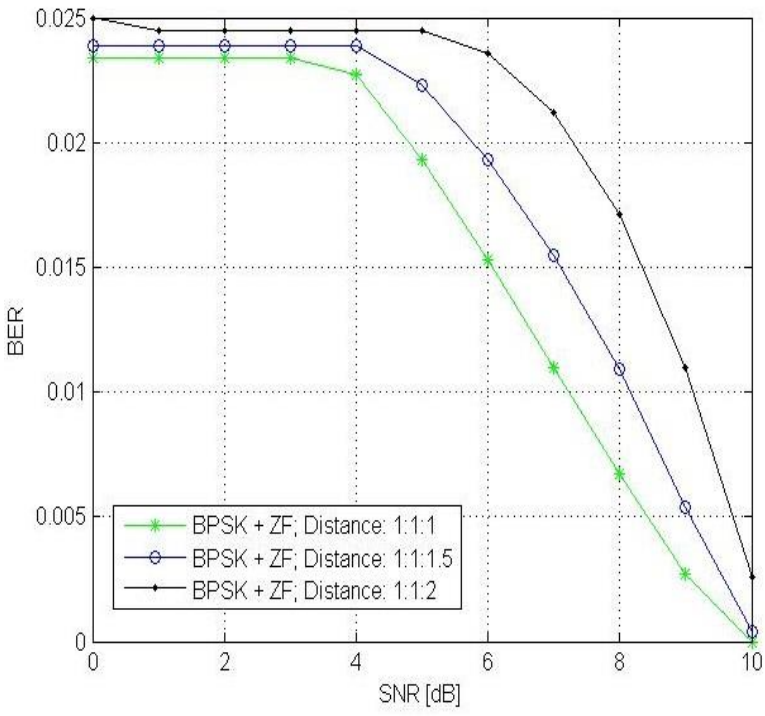

Fig. 2. BER performance of a convolutionally encoded and single relayed MIMO Cooperative OFDM wireless communication system with ZF aided signal detection, BPSK digital modulation and various distance between sender, relay and destination

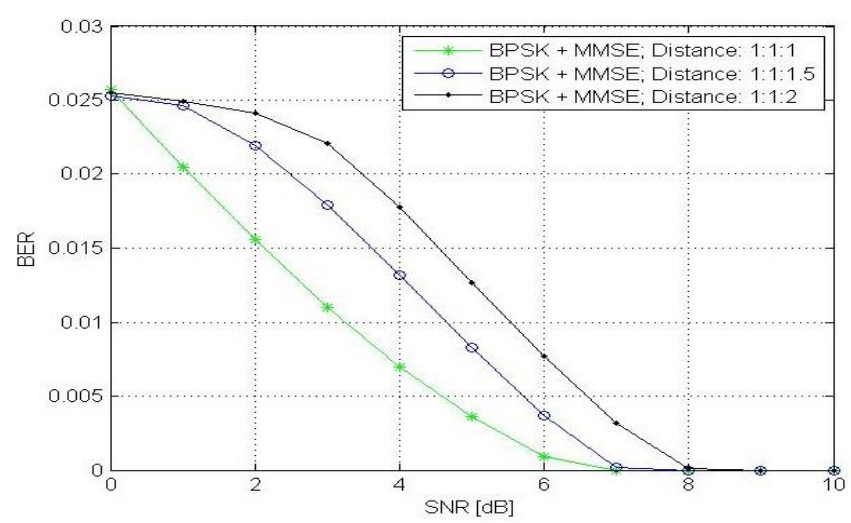

Fig. 3. BER performance of a convolutionally encoded and single relayed MIMO Cooperative OFDM wireless communication system with MMSE aided Signal detection and quadrature phase shift keying digital modulation schemes

In Fig. 3 under scenario of low order digital modulation and varying relative distances between sender, relay and destination we can have a clear picture on system performance enhancement. If the distance of separation among sender, relay and destination is identical then the performance improves significantly.

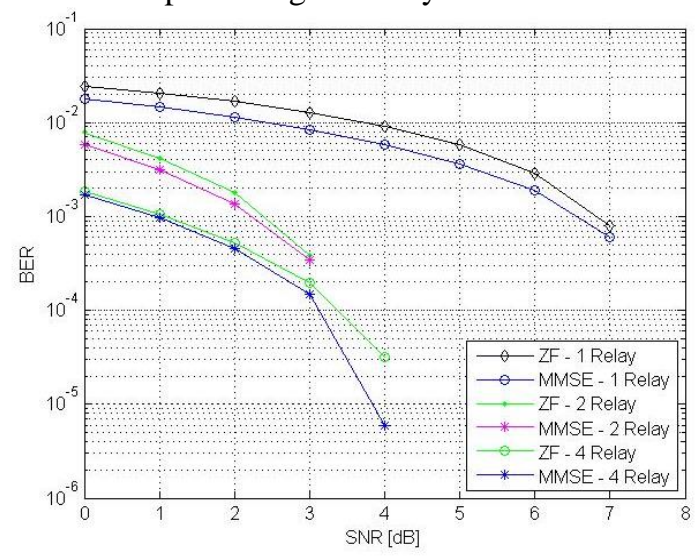

Fig. 4. BER performance comparison for different number of relays, ZF and MMSE signal detection schemes

In Fig. 4 we have compared the performance of the system by varying the number of relays in our proposed model (MIMO-OFDM and MIMO-OFDM with STBC) with two detection techniques. We observed that the system performance is better with four relays rather than using single or two relays. In case of single, two and four relays the MMSE receiver is better than $\mathrm{ZF}$ receiver. A signal to noise ratio (SNR) improvement of $1.2 \mathrm{~dB}$ and $1.4 \mathrm{~dB}$ in MMSE with 4 relays as compared to MMSE with 2 relays and ZF with 2 relays respectively when $0.001(0.1 \%)$ BER.

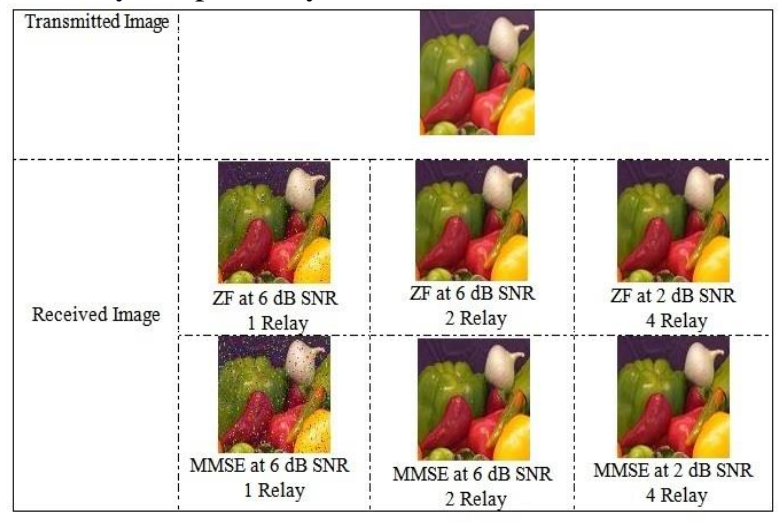

Fig. 5. Transmitted and Received Images for multiple relays, ZF and MMSE channel equalization schemes

In Fig. 5 we can see the transmitted and received colour images at typically assumed SNR values of $2 \mathrm{~dB}$ and $6 \mathrm{~dB}$ for the multiple relaying scheme aided cooperative MIMO OFDM system under implementation of ZF and MMSE channel equalization schemes. In case of four relay, the retrieved image is clearer than the single and two relays.

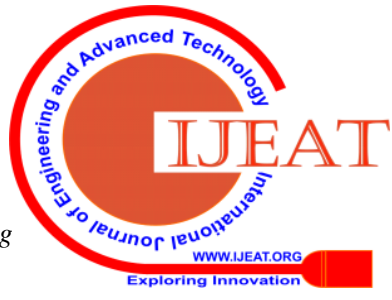




\section{CONCLUSION}

Cooperative MIMO_OFDM system with ZF and MMSE signal detection scheme aided by multiple relays has been presented in this article. The main purpose of this study is to find a suitable way to minimize the SNR and BER. By minimizing SNR and BER, communication with higher reliability with high speed can be achieved. We have observed that the performance of the proposed wireless communication system is better in AF relay with MMSE scheme as compared to ZF scheme. Additionally, in case of identical distances between source, relay and destination, the system shows better performance in both cases. We can also conclude that if we use higher number of relays, the color image retrieval becomes more satisfactory and decrease the SNR values.

\section{REFERENCES}

1. M. M. Rahman, M. S. Alam, S. Islam, M. A. A. Samy, \& N. A. Islam, "Cooperative MIMO OFDM system based on Amplify and Forward Relay: Evaluation of ZF-SIC and MMSE-SIC equalization," Przegląd Elektrotechniczny, vol. 1, no. 9, 2018, pp. 77-81.

2. P. Liu, Z. Tao, Z. Lin, E. Erkip, \& S. Panwar, "Advances in smart antennas - Cooperative wireless communications: a cross-layer approach," IEEE Wireless Commun., vol. 13, no. 4, 2006 pp. 84-92.

3. S. Wang \& J. Nie, "Energy Efficiency Optimization of Cooperative Communication in Wireless Sensor Networks," EURASIP Journal on Wireless Communications and Networking, vol. 1, Apr. 2010, pp. 1-8.

4. E. C. V. D. Meulen, "Three-terminal communication channels," Advances in Appl. Probability, vol.3 no. 1, 1971 pp. $120-154$.

5. T. Cover \& A. Gamal, "Capacity theorems for the relay channel," IEEE Trans. on Inf. Theory, vol. 25, no. 5, 1979, pp. 572-584.

6. M. Janani, A. Hedayat, T. Hunter \& A. Nosratinia, "Coded Cooperation in Wireless Communications: Space-Time Transmission and Iterative Decoding," IEEE Trans. on Signal Process., vol. 52, no. 2, 2004, pp. 362-371.

7. J. Laneman, D. Tse \& G. Wornell, "Cooperative Diversity in Wireless Networks: Efficient Protocols and Outage Behavior," IEEE Trans. on Inf. Theory, 50 no. 12, 2004, pp. 3062-3080.

8. T. Nechiporenko, K. Phan, C. Tellambura \& H. Nguyen, "On the capacity of Rayleigh fading cooperative systems under adaptive transmission," IEEE Trans. on Wireless Commun., vol. 8, no. 4, 2009, pp. 1626-1631.

9. V. Mahinthan, H. Rutagemwa, J. Mark \& X. Shen, "Cross-Layer Performance Study of Cooperative Diversity System With ARQ," IEEE Trans. on Veh. Technol., vol. 58, no. 2, 2009, pp. 705-719.

10. K.-S. Hwang, Y.-C. Ko \& M.-S. Alouini, "Performance analysis of incremental opportunistic relaying over identically and non-identically distributed cooperative paths," IEEE Trans. on Wireless Commun., vol. 8, no. 4, 2009, pp. 1953-1961.

11. J. Boyer, D. Falconer \& H. Yanikomeroglu, "Multihop Diversity in Wireless Relaying Channels," IEEE Trans. on Commun., vol. 52, no. 10, 2004, pp. 1820-1830.

12. D. Chen, \& J. Laneman, "Modulation and demodulation for cooperative diversity in wireless systems," IEEE Trans. on Wireless Commun., vol. 5, no. 7, 2006, pp. 1785-1794.

13. S. Sohaib \& D. K. So, "Energy allocation for green multiple relay cooperative communication,"EURASIP Journal on Wireless Communications and Networking, vol. 1, 2012, pp. 291-301.

14. M. M. Hossain \& S. E. Ullah, "Performance Evaluation of a Cooperative OFDM System with implementation of DAF and AAF Relaying Protocols on Color image transmission," Int. J. of Inf. Technol. Convergence and Services, vol. 1, no. 3, 2001, pp. 35-45.

15. N. S. Kumar \& K. R. S. Kumar, "Performance Analysis and Comparison of Zero - forcing SIC and MMSE SIC for MIMO Receivers using BSPK and 16- QAM Modulation methods," Int. J. of Comput. Sci. \& Eng. Technol., vol. 1, no. 8, 2011, pp. 530-533.

16. IEEE 802.16's Relay Task Group, http://www.ieee802.org/16/relay, Accessed: 04 Feb. 2020.

17. B. Can, M. Portalski, H. Lebreton, S. Frattasi, \& H. Suraweera, "Implementation Issues for OFDM-Based Multihop Cellular Networks," IEEE Commun. Mag. vol. 45, no. 9, 2007, pp. 74-81.

18. P. U. Sripathi and J. S. Lehnert, "A throughput scaling law for a class of wireless relay networks," Conf. Record of the Thirty-Eighth Asilomar
Conf. on Signals, Syst. and Comput., Pacific Grove, CA, USA, vol. 2 2004, pp. 1333-1337.

19. X. Tang and Y. Hua, "Optimal waveform design for MIMO relaying," IEEE 6th Workshop on Signal Process. Advances in Wireless Commun., New York, NY, USA., 2005, pp. 289-293.

20. Y.-W. P. Hong, W.-J. Huang \& C.-C. J. Kuo, "Cooperative Communications and Networking Technologies and System Design," New York, NY: Springer US., 2010.

21. Y. S. Cho, J. Kim, W. Y. Yang \& C. G. Kang, "Mimo-Ofdm wireless communications with Matlab," Singapore: IEEE Press, 2011.

22. L. Bai \& J. Choi, "Low complexity Mimo detection," Berlin: Springer, 2014.

\section{AUTHORS PROFILE}

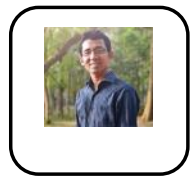

Md Mirazur Rahman, has accomplished M.Sc. from Queen Mary University of London in the field of Wireless Networks, in 2009. He received B.Sc. degree in Electrical, Electronic \& Communication engineering from Military Institute of Science \& technology, Dhaka, in 2007. He is currently working as an Assistant Professor, in the department of Electrical and Electronic Engineering, in Primeasia University, Dhaka. His research interests include smart and integrated antenna systems, MIMO, RFID and wireless sensor networks. He has published seven articles in international journals and conferences.

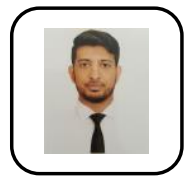

Md. Abdullah-Al-Mamun, received his B.Sc. degree in Electrical and Electronic Engineering from Ahsanullah University of Science \& Technology, Dhaka, Bangladesh, in 2018. He is currently working as a Lecturer in the Department of Electrical and Electronic Engineering in Ahsanullah University of Science \& Technology, Dhaka, Bangladesh. His research interests include Power Electronics and Demand Side Management.

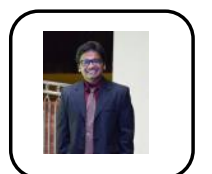

Rizal Fathoni Kabir, received his B.Sc. degree in Electrical, Electronic and Communication Engineering from Military Institute of Science and Technology, Dhaka, Bangladesh in 2015. He is currently working in National Electric BD as a Senior Executive. He has three years' experience in Telecommunication sector. His research interests are: Photonics, IP Networking, Optical Fiber Communication and MIMO.

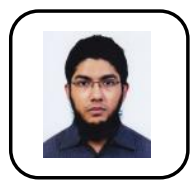

Abidur Rahman, received his B.Sc. and M.Sc. degree in Electrical and Electronic Engineering from Ahsanullah University of Science \& Technology, Dhaka, Bangladesh, in 2015 and 2019 respectively. He is currently serving as a Lecturer in the Department of Electrical and Electronic Engineering in Ahsanullah University of Science \& Technology, Dhaka, Bangladesh. His research interests include smart grid, demand side management, and electric vehicles.

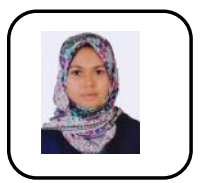

Subaha Mahmuda, was born in Mymensingh, Bangladesh in 1988. She has completed her M.Sc in the field of electronics and communication engineering from Izmir Institute of Technology, Turkey under Turkish Government scholarship in September, 2015. She has been working as a Lecturer in the Department of Electrical and Electronic Engineering at Primeasia University, Bangladesh for last 2.5 years. Ms. Mahmuda published eight papers in different international journals and conferences. 